\title{
Protein characteristics of chinese black-grained wheat
}

\author{
Wende $\mathrm{Li}^{\text {a }}$, Trust Beta ${ }^{\mathrm{b}, *}$, Shancheng Sun ${ }^{\mathrm{c}}$, Harold Corke ${ }^{\mathrm{d}}$ \\ ${ }^{a}$ Cereal Chemistry and Quality Analysis Laboratory, Faculty of Chemistry and Chemical Engineering, \\ Shanxi University, Taiyuan City 030006, PR China \\ ${ }^{\mathrm{b}}$ Department of Food Science, University of Manitoba, Winnipeg, Manitoba, Canada R3T 2N2 \\ ${ }^{\mathrm{c}}$ Shanxi Academy of Agricultural Science, Taiyuan City 030031, PR China \\ ${ }^{d}$ Cereal Science Laboratory, Department of Botany, University of Hong Kong, Hong Kong
}

Received 3 June 2005; received in revised form 24 June 2005; accepted 27 June 2005

\section{Abstract}

Protein properties of black-grained wheat (BGW) were compared with those of five carefully selected wheat controls (Taifen 1,

13 Klasic, Yecora Rojo, Glenlea and Anza) in order to find potential uses for BGW. Protein content, mixing properties, gluten index

14 and amino acid composition were measured. BGW whole meal had a higher protein content $(17.71 \%)$ than was found in controls.

15 Gluten index of BGW flour (69.74) was generally low compared to controls. Mid-line peak times determined using mixograph were

16 significantly longer $(p<0.05)$ for most controls $(5.41-6.27 \mathrm{~min})$ in comparison to BGW flour $(<3.00 \mathrm{~min})$. Dough stickiness (223.76 g) of BGW was somewhat stronger than that of Klasic and CES flours. Total essential amino acid and total amino acid contents in whole meal were $4.45 \%$ and $15.74 \%$, respectively, for BGW. The amino acid composition was relatively stable after high-temperature drying of wet BGW gluten. In vitro protein digestibility of BGW wheat meal was the lowest.

(c) 2005 Published by Elsevier Ltd.

\section{Introduction}

Black-coloured foods have a special place in Chinese food culture and enjoy wide acceptance in the marketplace. Many studies on black-seeded cereals have proven them to be associated with health and improved nutrition, and therefore form the basis for high value products, for instance, popular food products from black-grained rice and black-grained soybean (Lai, 1995; Lai \& Zhang, 1995). In fact, since the 1970s the Wheat Biotechnology Laboratory of the Institute of Crop Genetics, Shanxi Academy of Agricultural Science, has been engaged in research leading to the development of black-grained wheat from previously existing

\footnotetext{
${ }^{*}$ Corresponding author. Tel.: +1 204474 8214; fax: +1 204474 $7630 / 8214$.

E-mail address: trust_beta@umanitoba.ca (T. Beta).
}

blue and purple lines (Sun et al., 1996, 1999). After over 20 years effort a new black-grained wheat variety (BGW) has been developed and it is now available for utilization as a new raw food material for value-added products (Bai et al., 2000, 2002; Li, Sun, \& Ren, 2004; Yang, Li, Chu, \& Sun, 2001). Elemental Se content of BGW was high up to $1.04 \mathrm{mg} / \mathrm{kg}$ in comparison with $0.26 \mathrm{mg} / \mathrm{kg}$ of common wheat (Bai et al., 2000). Seed colour of BGW is visually black and the grain size is comparable to that of the controls chosen in the current investigation. The colour of wheat, usually white or red (although purple is known), is related to pigments in the seed coat. Basic wheat pigments include carotenes, xanthophylls and phenolic compounds (Beta, Nam, Dexter, \& Sapirstein, 2005; Kruger \& Reed, 1988). The main pigment component of BGW seed was an anthocyanin phenolic compound (Sun, Sun, \& Wang, 2004). Anthocyanins are known to exhibit good antioxidant activity 
54 (Awika, Rooney, \& Waniska, 2004). While there are 55 several chemical components in wheat, traditional nutri56 ents of major importance include starch and proteins. 57 Among cereals, only wheat has the ability to form a 58 strong, cohesive dough due to the uniqueness of its 59 proteins.

60 Evaluation of protein properties includes determina61 tion of amino acid composition, molecular weights, 62 protein digestibility, and gluten strength. Protein digest63 ibility is essentially a measure of the rate of in vitro pro64 tein hydrolysis by digestive enzymes. It is also a factor 65 most likely to affect amino acid availability since prote66 olysis is influenced both by the linear amino acid 67 sequence and the tertiary structure of a protein (Gopal, 68 Monteiro, Virupaksha, \& Ramachandra, 1988). Gluten 69 properties are associated with the end use of wheat flour. 70 The total gluten content and composition in the wheat 71 flour protein is of interest in the nutritional evaluation 72 of the wheat accessions (Abdel-Aal, Hucl, \& Sosulski, 73 1995). The gluten index is used as a measure of gluten 74 strength. The objective of the study was to determine 75 the protein properties of $\mathrm{BGW}$ and compare them to 76 five carefully selected wheat controls. The results will 77 be used to identify potential uses for BGW as raw mate78 rial for food production.

\section{2. Materials and methods}

\section{2.1. Materials}

81 The samples used for the study comprised one black82 grained wheat and five carefully selected commercial 83 wheat reference samples used in bread and noodle pro84 duction. Chinese black-grained wheat (BGW) and one 85 commercial reference Taifen 1 wheat (TW) samples were 86 obtained from Institute of Crop Genetics, Shanxi Acad87 emy of Agricultural Science, Taiyuan, China. Three 88 commercial US cultivars, Anza wheat (AW, California), 89 Klasic wheat (KW, California) and Yecora Rojo wheat 90 (YRW, California), were supplied by the University of 91 California, Davis. One commercial Canadian extra 92 strong wheat Glenlea (GW, Manitoba) sample was ob93 tained from Canada. All reference wheat samples with 94 the exception of TW and AW are used for breadmaking. TW and AW are used for noodle production.

Wheat flour was obtained by milling grain with a Quadrumat Junior laboratory mill (Brabender OHG, Duisberg, Germany). After separating wheat bran, wheat flour extraction rate ranged from $70 \%$ to $80 \%$. Wheat whole meal was prepared by milling wheat grain with a Cyclone sample mill (Udy Corp., Fort Collins, Colorado, USA). Wheat whole meal included flour and bran.

Freeze-dried (FD) gluten was obtained by handwashing the flour dough according to the method of
Qiu (1998). Wet gluten was immediately frozen in liquid nitrogen and freeze-dried. Main steps during hand-washing were: first making $100 \mathrm{~g}$ flour to dough by adding adequate water $(25-35 \mathrm{~mL}$ depending on the flour), resting the dough in a covered container for $2 \mathrm{~h}$, and finally washing dough in $2000 \mathrm{~mL}$ water for $15 \mathrm{~min}$ at room temperature to remove starch. The washings were repeated three times.

Wet gluten yield and gluten index were determined by the machine washing Method 38-12 of the AACC (1995). Preparation of wet gluten was according to the method of Perten (1990). Briefly $10 \mathrm{~g}$ flour was mixed for $20 \mathrm{~s}$ with $4.8 \mathrm{~mL}$ of $2 \% \mathrm{NaCl}$ solution, followed by washing for $5 \mathrm{~min}$ with $2 \% \mathrm{NaCl}$ solution at a flow rate of $50-60 \mathrm{~mL} / \mathrm{min}$ on a special $88-\mu \mathrm{m}$ sieve using a Perten Glutomatic Gluten Index machine (Perten Instruments AB, S-141 05 Huddinge, Sweden). Afterwards, the wet gluten piece was centrifuged at $6000 \mathrm{rpm}$ for $1 \mathrm{~min}$ on a special $600-\mu \mathrm{m}$ metallic sieve using a Perten Centrifuge 2015 machine (Perten Instruments AB, S-141 05 Huddinge, Sweden). Wet gluten samples obtained from both sides of the sieve after centrifugation were dried at $150{ }^{\circ} \mathrm{C}$ (high temperature drying) using a special Perten Glutork 2020 dryer. The gluten that remained on top of the sieve after centrifugation was labeled as high temperaturedried (HTD) gluten 1. The gluten that passed through the sieve was labeled as HTD gluten 2. Total protein content of the above samples was analyzed by the AACC Method 46-11A (1995).

\subsection{In vitro protein digestibility}

Pepsin (Pepsin porcine gastric mucosa, 800-2500 units/mg protein, Sigma Chemical Co., St. Louis, USA) and trypsin (Trypsin from bovine pancreas, $\geqslant 10,000$ BAEE units/mg protein, Sigma Chemical Co., St. Louis, USA) were used for in vitro protein digestibility (IVPD) studies. IVPD was determined by an improved method of Ramachandra, Virupaksha, and Shadaksharaswamy (1977) and Gopal et al. (1988). For pepsin, $50 \mathrm{mg}$ of whole meal or dry gluten samples were weighed into a series of test tubes and $5.0 \mathrm{~mL}$ of $0.075 \mathrm{~N} \mathrm{HCl}$ and $0.5 \mathrm{~mL}$ of pepsin solution $(2.0 \mathrm{mg} / \mathrm{mL})$ in $0.075 \mathrm{~N} \mathrm{HCl}$ were added to each tube. The tubes were incubated at $37^{\circ} \mathrm{C}$ and enzyme action was stopped at $30,60 \mathrm{~min}$ and $24 \mathrm{~h}$ by addition of $5 \mathrm{~mL}$ of $10 \%(\mathrm{w} / \mathrm{v})$ trichloroacetic acid (TCA). The reaction mixture was filtered through Whatman No. 1 filter paper, and the residue on the filter was washed with warm water. Nitrogen in the residue was estimated by the micro-Kjeldahl procedure (AACC Method 46-11A, 1995). For trypsin, the same IVPD procedure was conducted essentially as described for pepsin, except that incubation was in $0.1 \mathrm{M}$ phosphate buffer, $\mathrm{pH}$ 7.6. IVPD was obtained by calculating the
136 
160 difference between the amount of total nitrogen in the 161 sample before and after in vitro digestion with pepsin 162 or trypsin. Kjeldahl nitrogen was multiplied by the 163 factor 5.7 to obtain total protein.

\section{2.3. Amino acid analysis}

165 Amino acid composition of flour, whole meal and 166 gluten samples of black-grained wheat and the four 167 wheat references was determined using a Hitachi Ami168 no Acid Analyser, Model Hitachi 835-50 (Tokyo, 169 Japan). Preparation of hydrolyzate was according to 170 the method of Anjuma, Ahmada, Butta, Sheikhb, 171 and Pasha (2005). Sample (0.1 g) was hydrolyzed with $17210 \mathrm{~mL}$ of $6 \mathrm{~N} \mathrm{HCl}$ at $110^{\circ} \mathrm{C}$ for $22 \mathrm{~h}$. The hydrolyzate 173 was evaporated under vacuum at $60^{\circ} \mathrm{C}$ to remove $174 \mathrm{HCl}$. Then the hydrolyzate was dissolved in $5 \mathrm{~mL}$ of $1750.02 \mathrm{~N} \mathrm{HCl}$, centrifuged at $1000 \mathrm{rpm}$ and filtered to 176 remove the visible sediments. The supernatant $177(20 \mu \mathrm{L})$ was injected into the amino acid analyzer 178 for the determination of the amino acid composition 179 of each sample. The amino acids were separated on 180 a cation exchanger resin column $(150 \mathrm{~mm} \times 2.6 \mathrm{~mm}$ 181 i.d., No. 2619 resin) using sodium citric acid buffer 182 at $\mathrm{pH} 2.2$, a column temperature of $53^{\circ} \mathrm{C}$, a flow rate 183 of $0.225 \mathrm{~mL} \mathrm{~min}^{-1}$ and a postcolumn reaction with 184 ninhydrin $\left(0.3 \mathrm{~mL} \mathrm{~min}{ }^{-1}\right.$ ninhydrin flow rate) followed 185 by a photometric detection at $570 \mathrm{~nm}$ according to the 186 procedure of Llames and Fontaine (1994). Amino acid 187 standard (AAS18, Sigma Chemical Co., St. Louis, 188 USA) was used for calibration. Tryptophan was not 189 determined. Sample results were expressed as percent190 age amino acid composition on dry weight basis.

\section{2.4. Dough stickiness determination}

192 To measure dough stickiness, three doughs were 193 prepared from individual flours and the mean taken 194 as the stickiness value. A dough prepared from flour 195 (2 g, 14\% moisture basis) and measured water was 196 mixed to its optimum in a $2 \mathrm{~g}$ mixograph instrument 197 (TMCO, Lincoln, NE) and transferred into the Stick198 iness Cell (SMS/KSU, Haslemere, England). Dough 199 stickiness data was determined using a TA-XT2 tex200 ture analyser (Stable Micro Systems, Godalming, 201 UK) following the procedures described by Chen 202 and Hoseney (1995). Parameter selection of texture 203 analyzer was as follows: No. 3 TA-XT2 library pro204 gram, $40 \mathrm{~g}$ compression force, plexiglass probe of $20525 \mathrm{~mm}$ diameter, $5 \mathrm{~g}$ trigger force, probe compression 206 travel speed of $2 \mathrm{~mm} / \mathrm{s}$, probe reversing speed of $20710 \mathrm{~mm} / \mathrm{s}$, holding time of $0.1 \mathrm{~s}$, and probe travel dis208 tance of $4 \mathrm{~mm}$ depth. Four determinations were per209 formed per dough. The stickiness readings were 210 averaged as dough stickiness.

\subsection{Electrophoresis}

Laemmli's (1970) discontinuous high resolution sodium dodecylsulfate polyacrylamide gel electrophoresis (SDS-PAGE) system, as modified by Fullington, Cole, and Kasarda (1983) for wheat seed storage proteins, was used to extract and fractionate total proteins in $10 \%(\mathrm{w} / \mathrm{v})$ polyacrylamide gels. Electrophoresis was overnight at $8 \mathrm{~mA}$ constant current until the tracking dye, pyronin $\mathrm{Y}$ had reached the bottom of the gel. Molecular weight markers $(205,116,66$ and $39.8 \mathrm{kDa})$ were purchased from Sigma (F3526, Sigma Chemical Co., St. Louis, USA).

\subsection{Mixograph analysis}

Mixograph characteristics were determined using a National 2g Mixograph Instrument (TMCO, Lincoln, NE) following the procedures described by Rath, Gras, Wrigley, and Walker (1990) and in the AACC Method 54-40A (1995).

\subsection{Statistical analysis}

All samples were analyzed at least in duplicate determinations and the results were presented as averages. Data were subjected to analysis of variance (ANOVA). Means were separated using Fisher's protected least significant difference (LSD) test at $p=0.05$. Quantitative results were expressed on a dry weight basis (dwb), unless stated.

\section{Results and discussion}

\subsection{Gluten index and gluten content}

A high gluten index value indicated that limited wet gluten could pass through the special gluten index sieve after centrifugation. Taifen 1 wheat had the lowest gluten index value in five wheat cultivars (Table 1). Gluten index of Chinese black-grained wheat (BGW) was 69.74, a significantly higher value $(p<0.05)$ than that of Taifen 1 (TW), but significantly lower $(p<0.05)$ than that of Klasic (KW), Yecora Rojo (YRW) and Glenlea wheat $(\mathrm{GW})$. Low gluten index value also showed poor strength of wet gluten dough as observed in BGW flour in comparison to that of KW, YRW and GW flours. For breadmaking, the optimum gluten index range is between 60 and 90 (Perten, 1990). The results were consistent with our earlier experiment on SDS sedimentation values (SDS-SV) of their whole meals. The order of their SDS-sedimentation test results was as follows: GW $16.9 \mathrm{~mL} / \mathrm{g}>\mathrm{KW} \quad 16.5 \mathrm{~mL} / \mathrm{g}>\mathrm{YRW} \quad 15.0 \mathrm{~mL} / \mathrm{g}>$ BGW $13.3 \mathrm{~mL} / \mathrm{g}>\mathrm{TW} 9.9 \mathrm{~mL} / \mathrm{g}$ (Li, Corke, \& Sun, 
Table 1

Gluten index, wet gluten and dry gluten content of wheat flours

\begin{tabular}{llll}
\hline & $\mathrm{GI} \%$ & $\mathrm{WG} \%(14 \% \mathrm{mb})$ & $\mathrm{DG} \%(\mathrm{dwb})$ \\
\hline BGW-flour & $69.74 \mathrm{~b}$ & $41.96 \mathrm{~b}$ & $17.15 \mathrm{~b}$ \\
TW-flour & $50.09 \mathrm{c}$ & $54.20 \mathrm{a}$ & $23.35 \mathrm{a}$ \\
KW-flour & $98.66 \mathrm{a}$ & $29.45 \mathrm{dc}$ & $11.93 \mathrm{c}$ \\
YRW-flour & $98.88 \mathrm{a}$ & $26.82 \mathrm{~d}$ & $10.84 \mathrm{c}$ \\
GW-flour & $99.37 \mathrm{a}$ & $31.23 \mathrm{c}$ & $12.66 \mathrm{c}$ \\
LSD & 8.9814 & 7.5264 & 3.6354 \\
\hline
\end{tabular}

GI, gluten index; WG, wet gluten; DG, dry gluten; LSD, least significance difference at $p<0.05$ level of probability. Mean values for flour samples having similar letters in the same column are not significantly different.

257 1998). SDS-sedimentation value is often used to screen 258 for gluten strength in wheat cultivars and has a positive 259 correlation with gluten strength (Dick \& Quick, 1983). 260 Statistical analysis showed that there was a positive cor261 relation coefficient of about 0.9606 between gluten index and SDS-sedimentation value (Li et al., 1998). Yields (\%) of wet and dry gluten in BGW flour were $41.96 \%$ and $17.15 \%$ respectively, significantly lower $(p<0.05)$ than that of TW flour, but higher $(p<0.05)$ in comparison to that of GW, KW and YRW flours (Table 1).

\subsection{In vitro protein digestibility}

IVPD results of five wheat flours or their FD gluten using pepsin and trypsin are shown in Table 2. IVPD was increased with increase in incubation time. With pepsin, IVPD of BGW whole meal was the lowest at each incubation time compared to the other four whole meals. At 30 and $60 \mathrm{~min}$, BGW whole meal had significantly lower IVPD $(p<0.05)$ than the rest of the wheat whole meal. At $24 \mathrm{~h}$, only KW whole meal had significantly higher IVPD $(p<0.05)$ than BGW and other whole meal.

IVPD of BGW FD gluten was significantly the highest $(p<0.05)$ at $30 \mathrm{~min}$ and $24 \mathrm{~h}$ incubation time $(71.5 \%$ at $30 \mathrm{~min}, 96.4 \%$ at $24 \mathrm{~h}$ ). There were significant differences $(p<0.05)$ in IVPD of wheat FD gluten among some wheat cultivars at 30 and $60 \mathrm{~min}$, with the highest variation of $22.0 \%$ observed between BGW and GW FD gluten at $30 \mathrm{~min}$. But there were no significant differences $(p<0.05)$ in IVPD of wheat FD gluten among wheat cultivars at $24 \mathrm{~h}$.

In the case of trypsin, most of the digestion took place in $60 \mathrm{~min}$ for wheat whole meal. Further incubation up to $24 \mathrm{~h}$ resulted only in a slight increase in hydrolysis. Differences in IVPD among wheat cultivars were not significant after $1-24-\mathrm{h}$ periods of incubation. IVPD of BGW after 30 min incubation was significantly different $(p<0.05)$ from YRW and GW whole meal. However, incubation periods longer than $30 \mathrm{~min}$ resulted in similar IVPD among the whole meals. Results obtained for the IVPD of FD gluten showed significant differences $(p<0.05)$ among wheat cultivars. At 30 min incubation, IVPD of BGW FD gluten was significantly higher $(p<0.05)$ than all the glutens with the exception of YWR gluten. At $60 \mathrm{~min}$ and $24 \mathrm{~h}$ incubation, IVPD for BGW FD gluten was significantly higher $(p<0.05)$ compared to the four wheat gluten controls.

\subsection{Amino acid compositions}

Amino acid values and protein contents for blackgrained wheat and its controls are given in Table 3 (a-d). Generally similar levels of each type of amino acid were observed in BGW whole meal and its flour when compared to the four wheat controls. However, Val and Glu were slightly higher in BGW whole meal. A significant correlation between grain protein percentage and amino acid values has been reported (Acouistucci, Degidio, \& Vallega, 1995). A positive correlation between protein content and their total amino acid was also found. Total essential amino acid (TEAA) and total amino acid (TAA) contents were $4.45 \%$ and
280

281

282

283

284

285

286

287

288

289

290

291

292

293

294

295

296

297

298

299

300

301

302

303

304

305

306

307

308

309

310

311

312

313

314

315

Table 2

In vitro protein digestibility (IVPD): comparison of wheat whole meals and their FD gluten

\begin{tabular}{|c|c|c|c|c|c|c|}
\hline \multirow[t]{2}{*}{ Items } & \multicolumn{3}{|c|}{ IVPD $(\%)$ for pepsin } & \multicolumn{3}{|c|}{ IVPD $(\%)$ for trypsin } \\
\hline & $30(\min )$ & $60(\min )$ & $24(\mathrm{~h})$ & $30(\min )$ & $60(\min )$ & $24(\mathrm{~h})$ \\
\hline BGW-whole meal & $64.72 b$ & $67.65 b$ & $82.42 b$ & $60.16 \mathrm{a}$ & $66.42 \mathrm{a}$ & $66.87 \mathrm{a}$ \\
\hline TW-whole meal & $66.90 \mathrm{a}$ & $71.07 \mathrm{a}$ & $82.94 b$ & $60.70 \mathrm{a}$ & $64.93 \mathrm{a}$ & $66.62 \mathrm{a}$ \\
\hline $\mathrm{KW}$-whole meal & $66.16 \mathrm{a}$ & $71.11 \mathrm{a}$ & $87.23 \mathrm{a}$ & $59.86 \mathrm{ba}$ & $63.31 \mathrm{a}$ & $67.15 \mathrm{a}$ \\
\hline YRW-whole meal & $69.50 \mathrm{a}$ & $71.22 \mathrm{a}$ & $84.43 b$ & $57.81 \mathrm{~b}$ & $66.60 \mathrm{a}$ & $67.84 \mathrm{a}$ \\
\hline GW-whole meal & $67.75 \mathrm{a}$ & $69.59 \mathrm{ab}$ & $84.04 \mathrm{~b}$ & $58.04 \mathrm{~b}$ & $64.10 \mathrm{a}$ & $65.93 \mathrm{a}$ \\
\hline LSD & 3.4208 & 2.8920 & 2.5518 & 2.0649 & 3.7229 & 3.4873 \\
\hline BGW-FD-gluten & $71.52 \mathrm{a}$ & $78.15 b$ & $96.45 \mathrm{a}$ & $24.99 b$ & $45.30 \mathrm{a}$ & $58.58 \mathrm{a}$ \\
\hline TW-FD-gluten & $51.50 \mathrm{~d}$ & $59.80 \mathrm{~d}$ & $95.07 \mathrm{a}$ & $6.71 \mathrm{~d}$ & $7.12 \mathrm{e}$ & $53.69 \mathrm{~b}$ \\
\hline KW-FD-gluten & $66.75 b$ & $81.17 \mathrm{a}$ & $95.42 \mathrm{a}$ & $17.43 \mathrm{c}$ & $17.78 \mathrm{~d}$ & $36.55 \mathrm{e}$ \\
\hline YRW-FD-gluten & $64.01 \mathrm{c}$ & $62.53 \mathrm{c}$ & $95.37 \mathrm{a}$ & $35.22 \mathrm{a}$ & $39.40 \mathrm{~b}$ & $44.28 \mathrm{~d}$ \\
\hline GW-FD-gluten & $49.47 \mathrm{~d}$ & $61.90 \mathrm{dc}$ & $94.62 \mathrm{a}$ & $15.73 \mathrm{c}$ & $20.55 c$ & $47.02 \mathrm{c}$ \\
\hline LSD & 2.1415 & 2.516 & 2.1989 & 2.0913 & 2.3542 & 2.27217 \\
\hline
\end{tabular}

LSD, least significance difference at $p<0.05$ level of probability. Mean values for meal or gluten samples having similar letters in the same column are not significantly different. 
Table 3a

Amino acid comparison of whole meals (WM) $(\%, \mathrm{dwb})$

\begin{tabular}{|c|c|c|c|c|c|c|}
\hline Items & BGW-WM & TW-WM & KW-WM & YRW-WM & GW-WM & LSD \\
\hline ASP (\%) & $0.70 \mathrm{a}$ & $0.67 \mathrm{a}$ & $0.52 \mathrm{a}$ & $0.51 \mathrm{a}$ & $0.55 \mathrm{a}$ & 0.2135 \\
\hline $\mathrm{THR}^{\mathrm{a}}(\%)$ & $0.39 \mathrm{a}$ & $0.32 \mathrm{a}$ & $0.28 \mathrm{a}$ & $0.28 \mathrm{a}$ & $0.31 \mathrm{a}$ & 0.1382 \\
\hline SER $(\%)$ & $0.67 \mathrm{a}$ & $0.58 \mathrm{a}$ & $0.51 \mathrm{a}$ & $0.51 \mathrm{a}$ & $0.55 \mathrm{a}$ & 0.1841 \\
\hline GLU (\%) & $6.16 \mathrm{a}$ & $5.67 \mathrm{ba}$ & $4.78 b$ & $4.70 \mathrm{~b}$ & $4.85 b$ & 0.9851 \\
\hline GLY (\%) & $0.61 \mathrm{a}$ & $0.56 \mathrm{a}$ & $0.49 \mathrm{a}$ & $0.49 \mathrm{a}$ & $0.50 \mathrm{a}$ & 0.1526 \\
\hline ALA $(\%)$ & $0.52 \mathrm{a}$ & $0.48 \mathrm{a}$ & $0.39 a$ & $0.39 \mathrm{a}$ & $0.42 \mathrm{a}$ & 0.1369 \\
\hline CYS (\%) & $0.13 \mathrm{a}$ & $0.07 \mathrm{a}$ & $0.09 \mathrm{a}$ & $0.09 \mathrm{a}$ & $0.11 \mathrm{a}$ & 0.0841 \\
\hline $\mathrm{VAL}^{\mathrm{a}}(\%)$ & $0.68 \mathrm{a}$ & $0.60 \mathrm{ba}$ & $0.49 \mathrm{~b}$ & $0.49 \mathrm{~b}$ & $0.52 b$ & 0.1491 \\
\hline MET (\%) & $0.19 \mathrm{a}$ & $0.11 \mathrm{ba}$ & $0.12 \mathrm{ba}$ & $0.06 \mathrm{~b}$ & $0.14 \mathrm{ba}$ & 0.1036 \\
\hline $\operatorname{ILE}^{\mathrm{a}}(\%)$ & $0.49 \mathrm{a}$ & $0.43 \mathrm{a}$ & $0.34 \mathrm{a}$ & $0.35 \mathrm{a}$ & $0.36 \mathrm{a}$ & 0.1572 \\
\hline $\operatorname{LEU}^{\mathrm{a}}(\%)$ & $1.01 \mathrm{a}$ & $0.99 a$ & $0.81 \mathrm{a}$ & $0.81 \mathrm{a}$ & $0.84 \mathrm{a}$ & 0.5021 \\
\hline $\mathrm{TYR}^{\mathrm{a}}(\%)$ & $0.37 \mathrm{a}$ & $0.17 \mathrm{a}$ & $0.29 \mathrm{a}$ & $0.31 \mathrm{a}$ & $0.28 \mathrm{a}$ & 0.2613 \\
\hline $\operatorname{PHE}^{\mathrm{a}}(\%)$ & $0.70 \mathrm{a}$ & $0.63 \mathrm{a}$ & $0.53 \mathrm{a}$ & $0.53 \mathrm{a}$ & $0.54 \mathrm{a}$ & 0.3382 \\
\hline LYS $^{\mathrm{a}}(\%)$ & $0.40 \mathrm{a}$ & $0.36 \mathrm{a}$ & $0.30 \mathrm{a}$ & $0.30 \mathrm{a}$ & $0.32 \mathrm{a}$ & 0.2085 \\
\hline $\operatorname{HIS}^{\mathrm{a}}(\%)$ & $0.32 \mathrm{a}$ & $0.30 \mathrm{a}$ & $0.24 \mathrm{a}$ & $0.24 \mathrm{a}$ & $0.25 \mathrm{a}$ & 0.1066 \\
\hline ARG $(\%)$ & $0.77 \mathrm{a}$ & $0.54 \mathrm{a}$ & $0.56 \mathrm{a}$ & $0.58 \mathrm{a}$ & $0.59 \mathrm{a}$ & 0.2692 \\
\hline PRO $(\%)$ & $1.55 \mathrm{a}$ & $1.44 \mathrm{a}$ & $1.19 \mathrm{a}$ & $1.18 \mathrm{a}$ & $1.18 \mathrm{a}$ & 0.4381 \\
\hline TEAAs ${ }^{\mathrm{b}}(\%)$ & $4.45 \mathrm{a}$ & $3.80 \mathrm{ba}$ & $3.28 \mathrm{~b}$ & $3.31 \mathrm{~b}$ & $3.42 b$ & 0.9014 \\
\hline $\mathrm{TAAs}^{\mathrm{c}}(\%)$ & $15.74 \mathrm{a}$ & $13.91 \mathrm{~b}$ & $11.94 \mathrm{c}$ & $11.81 \mathrm{c}$ & $12.28 \mathrm{c}$ & 1.4721 \\
\hline Protein $(\%)$ & $17.71 \mathrm{a}$ & $17.00 \mathrm{a}$ & $14.07 \mathrm{~b}$ & $13.67 \mathrm{~b}$ & $14.52 \mathrm{~b}$ & 1.6035 \\
\hline
\end{tabular}

LSD, least significance difference at $p<0.05$ level of probability. Mean values for amino acid or protein content having similar letters in the same row are not significantly different.

${ }^{\mathrm{a}}$ Essential amino acid.

b Total essential amino acid.

c Total amino acid.

$31615.74 \%$, respectively, for BGW whole meal. The TEAA 317 and TAA of BGW whole meal were significantly higher $318(p<0.05)$ than that of TW, KW, YRW and GW. BGW 319 flour contained $4.23 \%$ of TEAA and $15.54 \%$ of TAA 320 (Table 4). The TEAA of BGW flour was $21.55 \%$,
$36.45 \%, 54.38 \%$ and $29.75 \%$ higher than that of TW, 321 KW, YRW and GW, respectively. Similarly the TAA 322 of BGW flour was $18.35 \%, 33.62 \%, 52.80 \%$ and 323 $28.43 \%$ higher than that of TW, KW, YRW and GW, 324 respectively.

Table $3 b$

Amino acid comparison of flours $(\%, \mathrm{dwb})$

\begin{tabular}{|c|c|c|c|c|c|c|}
\hline Items & BGW-flour & TW-flour & KW-flour & YRW-flour & GW-flour & LSD \\
\hline ASP (\%) & $0.55 \mathrm{a}$ & $0.47 \mathrm{a}$ & $0.43 \mathrm{a}$ & $0.34 \mathrm{a}$ & $0.44 \mathrm{a}$ & 0.2514 \\
\hline $\mathrm{THR}^{\mathrm{a}}(\%)$ & $0.36 \mathrm{a}$ & $0.28 \mathrm{a}$ & $0.26 \mathrm{a}$ & $0.22 \mathrm{a}$ & $0.28 \mathrm{a}$ & 0.1836 \\
\hline SER $(\%)$ & $0.65 a$ & $0.54 \mathrm{a}$ & $0.49 a$ & $0.42 \mathrm{a}$ & $0.52 \mathrm{a}$ & 0.2671 \\
\hline GLU (\%) & $6.72 a$ & $5.87 \mathrm{ba}$ & $5.08 \mathrm{~b}$ & $4.55 \mathrm{cb}$ & $5.21 \mathrm{~b}$ & 1.1323 \\
\hline GLY (\%) & $0.50 \mathrm{a}$ & $0.41 \mathrm{a}$ & $0.41 \mathrm{a}$ & $0.33 \mathrm{a}$ & $0.43 \mathrm{a}$ & 0.1826 \\
\hline ALA (\%) & $0.41 \mathrm{a}$ & $0.32 \mathrm{a}$ & $0.30 \mathrm{a}$ & $0.25 \mathrm{a}$ & $0.33 \mathrm{a}$ & 0.1725 \\
\hline CYS (\%) & $0.16 \mathrm{a}$ & $0.11 \mathrm{a}$ & $0.08 \mathrm{a}$ & $0.06 \mathrm{a}$ & $0.11 \mathrm{a}$ & 0.1051 \\
\hline $\mathrm{VAL}^{\mathrm{a}}(\%)$ & $0.63 \mathrm{a}$ & $0.51 \mathrm{a}$ & $0.46 \mathrm{a}$ & $0.40 \mathrm{a}$ & $0.48 \mathrm{a}$ & 0.2471 \\
\hline MET (\%) & $0.19 \mathrm{a}$ & $0.14 \mathrm{a}$ & $0.14 \mathrm{a}$ & $0.11 \mathrm{a}$ & $0.15 \mathrm{a}$ & 0.1024 \\
\hline $\operatorname{ILE}^{\mathrm{a}}(\%)$ & $0.49 \mathrm{a}$ & $0.40 \mathrm{a}$ & $0.33 \mathrm{a}$ & $0.29 \mathrm{a}$ & $0.36 \mathrm{a}$ & 0.2252 \\
\hline $\operatorname{LEU}^{\mathrm{a}}(\%)$ & $1.07 \mathrm{a}$ & $0.88 \mathrm{ba}$ & $0.76 \mathrm{ba}$ & $0.69 b$ & $0.81 \mathrm{ba}$ & 0.3456 \\
\hline $\mathrm{TYR}^{\mathrm{a}}(\%)$ & $0.39 a$ & $0.33 \mathrm{a}$ & $0.30 \mathrm{a}$ & $0.26 \mathrm{a}$ & $0.32 \mathrm{a}$ & 0.1458 \\
\hline $\operatorname{PHE}^{\mathrm{a}}(\%)$ & $0.70 \mathrm{a}$ & $0.60 \mathrm{a}$ & $0.53 \mathrm{a}$ & $0.48 \mathrm{a}$ & $0.54 \mathrm{a}$ & 0.2581 \\
\hline LYS $^{\mathrm{a}}(\%)$ & $0.29 \mathrm{a}$ & $0.24 \mathrm{a}$ & $0.24 \mathrm{a}$ & $0.20 \mathrm{a}$ & $0.25 \mathrm{a}$ & 0.1106 \\
\hline $\operatorname{HIS}^{\mathrm{a}}(\%)$ & $0.30 \mathrm{a}$ & $0.24 \mathrm{a}$ & $0.22 \mathrm{a}$ & $0.20 \mathrm{a}$ & $0.22 \mathrm{a}$ & 0.1257 \\
\hline ARG (\%) & $0.64 \mathrm{a}$ & $0.49 \mathrm{a}$ & $0.48 \mathrm{a}$ & $0.39 \mathrm{a}$ & $0.49 \mathrm{a}$ & 0.2891 \\
\hline PRO (\%) & $1.52 \mathrm{a}$ & $1.32 \mathrm{a}$ & $1.11 \mathrm{a}$ & $0.99 \mathrm{~b}$ & $1.17 \mathrm{a}$ & 0.4713 \\
\hline TEAAs $^{\mathrm{b}}(\%)$ & $4.23 \mathrm{a}$ & $3.48 \mathrm{~b}$ & $3.10 \mathrm{~b}$ & $2.74 b$ & $3.26 \mathrm{~b}$ & 0.7016 \\
\hline $\mathrm{TAAs}^{\mathrm{c}}(\%)$ & $15.54 \mathrm{a}$ & $13.13 \mathrm{ba}$ & $11.63 b$ & $10.17 \mathrm{c}$ & $12.10 \mathrm{~b}$ & 2.1359 \\
\hline Protein $(\%)$ & $18.26 \mathrm{a}$ & $15.94 \mathrm{ba}$ & $13.76 \mathrm{~b}$ & $12.59 \mathrm{c}$ & $14.23 b$ & 2.6801 \\
\hline
\end{tabular}

LSD, least significance difference at $p<0.05$ level of probability. Mean values for amino acid or protein content having similar letters in the same row are not significantly different.

${ }^{\text {a }}$ Essential amino acid.

b Total essential amino acid.

${ }^{c}$ Total amino acid. 
Table 3c

Amino acid comparison of freeze-dried gluten (FDG) $(\%, \mathrm{dwb})$

\begin{tabular}{|c|c|c|c|c|c|c|}
\hline Items & BGW-FDG & TW-FDG & KW-FDG & YRW-FDG & GW-FDG & LSD \\
\hline $\operatorname{ASP}(\%)$ & $2.51 \mathrm{a}$ & $2.43 \mathrm{a}$ & $2.07 \mathrm{a}$ & $2.38 \mathrm{a}$ & $2.44 \mathrm{a}$ & 0.5013 \\
\hline $\operatorname{THR}^{\mathrm{a}}(\%)$ & $1.91 \mathrm{a}$ & $1.78 \mathrm{a}$ & $1.72 \mathrm{a}$ & $1.87 \mathrm{a}$ & $1.91 \mathrm{a}$ & 0.3152 \\
\hline SER $(\%)$ & $3.70 \mathrm{a}$ & $3.58 \mathrm{a}$ & $3.40 \mathrm{a}$ & $3.72 \mathrm{a}$ & $3.83 \mathrm{a}$ & 0.6051 \\
\hline GLU $(\%)$ & $37.01 \mathrm{a}$ & $36.92 \mathrm{a}$ & $33.18 \mathrm{~b}$ & $37.10 \mathrm{a}$ & $37.73 \mathrm{a}$ & 3.4018 \\
\hline GLY (\%) & $2.65 \mathrm{a}$ & $2.51 \mathrm{a}$ & $2.47 \mathrm{a}$ & $2.82 \mathrm{a}$ & $2.50 \mathrm{a}$ & 0.5043 \\
\hline ALA (\%) & $2.00 \mathrm{a}$ & $1.93 \mathrm{a}$ & $1.77 \mathrm{a}$ & $1.96 \mathrm{a}$ & $1.89 \mathrm{a}$ & 0.3815 \\
\hline CYS (\%) & $1.15 \mathrm{a}$ & $1.10 \mathrm{a}$ & $1.01 \mathrm{a}$ & $1.01 \mathrm{a}$ & $1.20 \mathrm{a}$ & 0.2306 \\
\hline VAL $^{\mathrm{a}}(\%)$ & $2.97 \mathrm{a}$ & $2.90 \mathrm{a}$ & $2.57 \mathrm{a}$ & $2.84 \mathrm{a}$ & $2.95 \mathrm{a}$ & 0.5611 \\
\hline MET $(\%)$ & $1.00 \mathrm{a}$ & $1.02 \mathrm{a}$ & $0.95 \mathrm{a}$ & $1.02 \mathrm{a}$ & $1.10 \mathrm{a}$ & 0.3029 \\
\hline $\operatorname{ILE}^{\mathrm{a}}(\%)$ & $2.68 \mathrm{a}$ & $2.61 \mathrm{a}$ & $2.28 \mathrm{a}$ & $2.53 \mathrm{a}$ & $2.60 \mathrm{a}$ & 0.5851 \\
\hline $\operatorname{LEU}^{\mathrm{a}}(\%)$ & $5.64 \mathrm{a}$ & $5.57 \mathrm{a}$ & $4.94 \mathrm{a}$ & $5.47 \mathrm{a}$ & $5.50 \mathrm{a}$ & 1.5025 \\
\hline TYR $^{\mathrm{a}}(\%)$ & $2.08 \mathrm{a}$ & $2.30 \mathrm{a}$ & $2.07 \mathrm{a}$ & $2.34 \mathrm{a}$ & $2.42 \mathrm{a}$ & 0.4705 \\
\hline $\operatorname{PHE}^{\mathrm{a}}(\%)$ & $3.91 \mathrm{a}$ & $3.78 \mathrm{a}$ & $3.26 \mathrm{a}$ & $3.82 \mathrm{a}$ & $3.98 \mathrm{a}$ & 0.8012 \\
\hline $\operatorname{LYS}^{\mathrm{a}}(\%)$ & $1.35 \mathrm{a}$ & $1.26 \mathrm{a}$ & $1.08 \mathrm{a}$ & $1.30 \mathrm{a}$ & $1.22 \mathrm{a}$ & 0.3476 \\
\hline $\operatorname{HIS}^{\mathrm{a}}(\%)$ & $1.40 \mathrm{a}$ & $1.39 \mathrm{a}$ & $1.21 \mathrm{a}$ & $1.40 \mathrm{a}$ & $1.41 \mathrm{a}$ & 0.3239 \\
\hline ARG $(\%)$ & $2.98 \mathrm{a}$ & $2.80 \mathrm{a}$ & $2.69 \mathrm{a}$ & $3.03 \mathrm{a}$ & $3.10 \mathrm{a}$ & 0.5317 \\
\hline PRO (\%) & $9.32 \mathrm{a}$ & $9.24 \mathrm{a}$ & $7.86 \mathrm{~b}$ & $9.04 \mathrm{ba}$ & $9.26 \mathrm{a}$ & 1.3602 \\
\hline TEAAs $^{\mathrm{b}}(\%)$ & $21.94 \mathrm{a}$ & $21.59 \mathrm{a}$ & $19.13 b$ & $21.57 \mathrm{a}$ & $21.99 \mathrm{a}$ & 2.3058 \\
\hline $\mathrm{TAAs}^{\mathrm{c}}(\%)$ & $84.28 \mathrm{a}$ & $83.12 \mathrm{a}$ & $74.53 b$ & $83.66 \mathrm{a}$ & $85.04 \mathrm{a}$ & 4.5605 \\
\hline Protein $(\%)$ & $86.44 \mathrm{a}$ & $84.69 \mathrm{a}$ & $77.94 b$ & $86.31 \mathrm{a}$ & $86.89 \mathrm{a}$ & 4.2062 \\
\hline
\end{tabular}

LSD, least significance difference at $p<0.05$ level of probability. Mean values for amino acid or protein content having similar letters in the same row are not significantly different.

${ }^{a}$ Essential amino acid.

b Total essential amino acid.

c Total amino acid.

326 Amino acid composition of FD and HTD gluten was 327 also compared (Tables $3 \mathrm{c}$ and 3d). HTD gluten was the 328 samples obtained by drying with Perten Glutork 2020

329 dryer at $150^{\circ} \mathrm{C}$. The amino acid composition between

330 FD and HTD glutens was useful in understanding the effect of temperature on gluten quality. The TEAA, TAA and protein contents ranged from $19.93 \%$ to $21.99 \%, 74.53 \%$ to $85.04 \%$ and $77.94 \%$ to $86.89 \%$, respectively, for the five FD gluten samples. High temperature drying of gluten resulted in significant

Table 3d

Amino acid comparison of high temperature dried gluten (HTDG) $(\%, \mathrm{dwb})$

\begin{tabular}{|c|c|c|c|c|c|c|c|c|}
\hline Items & BGW-HTDG 1 & BGW-HTDG 2 & TW-HTDG 1 & TW-HTDG 2 & KW-HTDG & YRW-HTDG & GW-HTDG & LSD \\
\hline ASP $(\%)$ & $2.29 \mathrm{a}$ & $2.24 \mathrm{a}$ & $1.59 \mathrm{~b}$ & $1.43 \mathrm{c}$ & $0.91 \mathrm{dc}$ & $2.22 \mathrm{a}$ & $2.03 \mathrm{ba}$ & 0.5516 \\
\hline $\mathrm{THR}^{\mathrm{a}}(\%)$ & $1.93 \mathrm{a}$ & $1.88 \mathrm{a}$ & $1.22 \mathrm{~b}$ & $1.08 \mathrm{~b}$ & $0.78 b$ & $1.95 \mathrm{a}$ & $1.90 \mathrm{a}$ & 0.5425 \\
\hline SER $(\%)$ & $3.83 \mathrm{a}$ & $3.74 \mathrm{a}$ & $2.60 \mathrm{~b}$ & $2.31 \mathrm{~b}$ & $1.75 b$ & $4.13 \mathrm{a}$ & $4.09 \mathrm{a}$ & 0.8613 \\
\hline GLU $(\%)$ & $38.64 \mathrm{a}$ & $37.46 \mathrm{a}$ & $26.42 \mathrm{a}$ & $23.68 \mathrm{a}$ & $18.60 \mathrm{~b}$ & $41.11 \mathrm{a}$ & $38.95 \mathrm{a}$ & 3.0572 \\
\hline GLY (\%) & $2.30 \mathrm{a}$ & $2.24 \mathrm{a}$ & $1.61 \mathrm{~b}$ & $1.45 \mathrm{~b}$ & $0.29 \mathrm{c}$ & $2.41 \mathrm{a}$ & $2.39 \mathrm{a}$ & 0.5081 \\
\hline ALA (\%) & $1.89 \mathrm{a}$ & $1.85 \mathrm{a}$ & $1.34 \mathrm{~b}$ & $1.22 \mathrm{~b}$ & $0.86 \mathrm{c}$ & $1.73 \mathrm{a}$ & $1.73 \mathrm{a}$ & 0.3503 \\
\hline CYS (\%) & $1.03 \mathrm{a}$ & $1.10 \mathrm{a}$ & $0.71 \mathrm{~b}$ & $0.64 b$ & $0.44 \mathrm{cb}$ & $0.86 \mathrm{ba}$ & $0.79 \mathrm{ba}$ & 0.3133 \\
\hline VAL $^{\mathrm{a}}(\%)$ & $3.01 \mathrm{a}$ & $2.91 \mathrm{a}$ & $2.07 \mathrm{~b}$ & $1.88 \mathrm{cb}$ & $1.36 \mathrm{c}$ & $2.77 \mathrm{a}$ & $2.65 \mathrm{a}$ & 0.5601 \\
\hline MET $(\%)$ & $1.17 \mathrm{a}$ & $1.13 \mathrm{a}$ & $0.74 b$ & $0.65 b$ & $0.21 \mathrm{c}$ & $1.13 \mathrm{a}$ & $1.10 \mathrm{a}$ & 0.2813 \\
\hline $\operatorname{ILE}^{\mathrm{a}}(\%)$ & $2.70 \mathrm{a}$ & $2.61 \mathrm{a}$ & $1.86 \mathrm{~b}$ & $1.68 b$ & $1.22 \mathrm{c}$ & $2.46 \mathrm{a}$ & $2.37 \mathrm{a}$ & 0.4561 \\
\hline $\operatorname{LEU}^{\mathrm{a}}(\%)$ & $5.68 \mathrm{a}$ & $5.52 \mathrm{a}$ & $3.92 \mathrm{~b}$ & $3.56 \mathrm{cb}$ & $2.61 \mathrm{c}$ & $5.55 \mathrm{a}$ & $5.29 \mathrm{a}$ & 1.0581 \\
\hline $\mathrm{TYR}^{\mathrm{a}}(\%)$ & $2.38 \mathrm{a}$ & $2.29 \mathrm{a}$ & $1.64 \mathrm{~b}$ & $1.50 \mathrm{~b}$ & $1.04 \mathrm{c}$ & $2.26 \mathrm{a}$ & $2.17 \mathrm{a}$ & 0.4501 \\
\hline $\operatorname{PHE}^{\mathrm{a}}(\%)$ & $3.98 \mathrm{a}$ & $3.87 \mathrm{a}$ & $2.78 \mathrm{bc}$ & $2.50 \mathrm{bc}$ & $1.93 \mathrm{c}$ & $4.32 \mathrm{a}$ & $3.82 \mathrm{a}$ & 0.8625 \\
\hline LYS $^{\mathrm{a}}(\%)$ & $1.02 \mathrm{a}$ & $1.00 \mathrm{a}$ & $0.72 \mathrm{cb}$ & $0.63 \mathrm{cb}$ & $0.48 \mathrm{c}$ & $1.09 \mathrm{a}$ & $0.97 \mathrm{a}$ & 0.2458 \\
\hline HIS $^{\mathrm{a}}(\%)$ & $1.43 \mathrm{a}$ & $1.37 \mathrm{a}$ & $0.97 \mathrm{~b}$ & $0.87 \mathrm{cb}$ & $0.66 \mathrm{c}$ & $1.43 \mathrm{a}$ & $1.29 \mathrm{a}$ & 0.2281 \\
\hline ARG $(\%)$ & $2.90 \mathrm{a}$ & $2.82 \mathrm{a}$ & $1.91 \mathrm{~b}$ & $1.72 \mathrm{cb}$ & $1.25 \mathrm{c}$ & $2.90 \mathrm{a}$ & $2.80 \mathrm{a}$ & 0.6012 \\
\hline PRO (\%) & $9.82 \mathrm{a}$ & $9.44 a$ & $6.56 \mathrm{~b}$ & $5.95 \mathrm{~b}$ & $4.43 c$ & $10.38 \mathrm{a}$ & $9.95 \mathrm{a}$ & 1.5305 \\
\hline TEAAs $^{\mathrm{b}}(\%)$ & $22.13 \mathrm{a}$ & $21.45 \mathrm{a}$ & $15.18 \mathrm{~b}$ & $13.70 \mathrm{~b}$ & $10.08 \mathrm{c}$ & $21.83 \mathrm{a}$ & $20.46 a$ & 2.8135 \\
\hline $\mathrm{TAAs}^{\mathrm{c}}(\%)$ & $86.00 \mathrm{a}$ & $83.47 \mathrm{a}$ & $58.65 \mathrm{~b}$ & $52.75 \mathrm{c}$ & $38.82 \mathrm{~d}$ & $88.72 \mathrm{a}$ & $84.29 \mathrm{a}$ & 5.0126 \\
\hline Protein $(\%)$ & $88.47 \mathrm{a}$ & $88.47 \mathrm{a}$ & $85.32 \mathrm{a}$ & $85.32 \mathrm{a}$ & $78.25 \mathrm{~b}$ & $90.81 \mathrm{a}$ & $86.73 a$ & 5.8206 \\
\hline
\end{tabular}

LSD, least significance difference at $p<0.05$ level of probability. Mean values for amino acid or protein content having similar letters in the same row are not significantly different. (Note. KW, YRW, GW yielded only gluten 1).

a Essential amino acid.

b Total essential amino acid.

c Total amino acid. 
336 decreases $(p<0.05)$ in amino acid content of some culti337 vars. For example, TAA content of KW HTD gluten 338 was only $38.82 \%$ in comparison with $74.53 \%$ of $\mathrm{KW}$ 339 FD gluten. Since the level of protein in the gluten dough 340 for KW was also low when compared to other cultivars 341 (Tables 3c and 3d), this indicated the presence of non342 protein components. At $150{ }^{\circ} \mathrm{C}$ it may be possible that 343 protein got bound or reacted with these non-protein 344 components and did not get fully hydrolyzed under 345 the conditions provided for hydrolysis to release amino 346 acids $\left(6 \mathrm{~N} \mathrm{HCl}, 110^{\circ} \mathrm{C}, 22 \mathrm{~h}\right)$. New high molecular 347 weight components were formed after preheating whey 348 protein isolate at $120^{\circ} \mathrm{C}$ for $3 \mathrm{~h}$, and $\beta$-lactoglobulin 349 was more sensitive to preheating than $\alpha$-lactalbumin, and preheating also resulted in the conformational 350 changes of proteins of whey protein isolate (Fujino et al., 1995). After high temperature drying, the order of TAA content was as follows: YRW HTD gluten $>$ BGW HTD gluten $1>$ GW HTD gluten $>$ TW HTD gluten $1>$ KW HTD gluten (Table 6). The TEAA and TAA content of HTD gluten 2 was similar or slightly lower in comparison with its HTD gluten 1 counterpart, however, the TAA content between TW HTD gluten 1 and TW HTD gluten 2 was significant $(p<0.05)$. The results also indicated that high temperature drying significantly $(p<0.05)$ reduced the TEAA and TAA amino acid contents of wet gluten dough for TW and KW (Tables $3 \mathrm{c}$ and $3 \mathrm{~d}$ ).

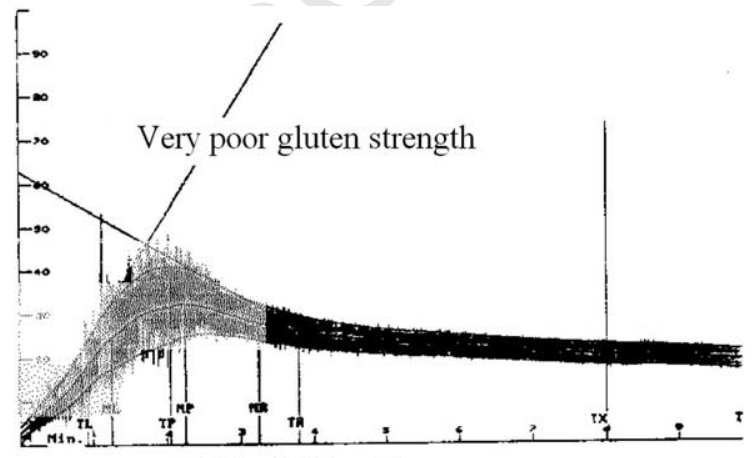

Taifen 1 Wheat Flour

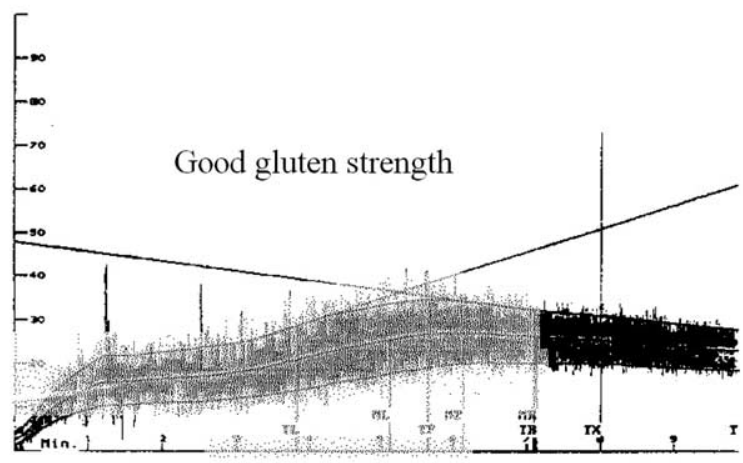

Yecora Rojo Wheat Flour

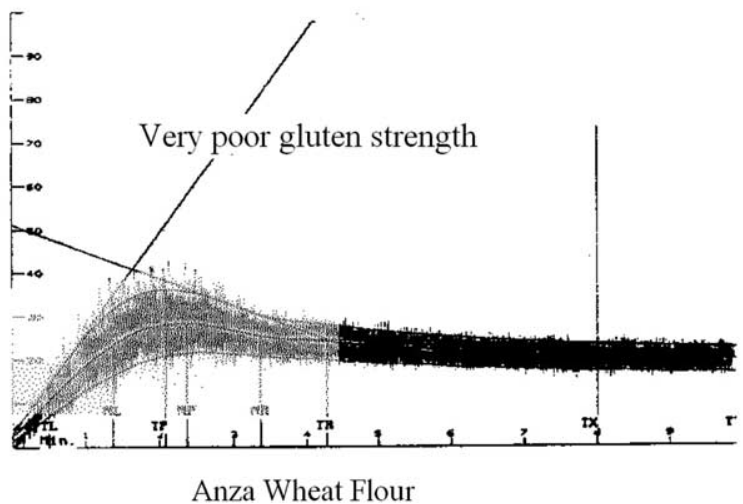

Anza Wheat Flour

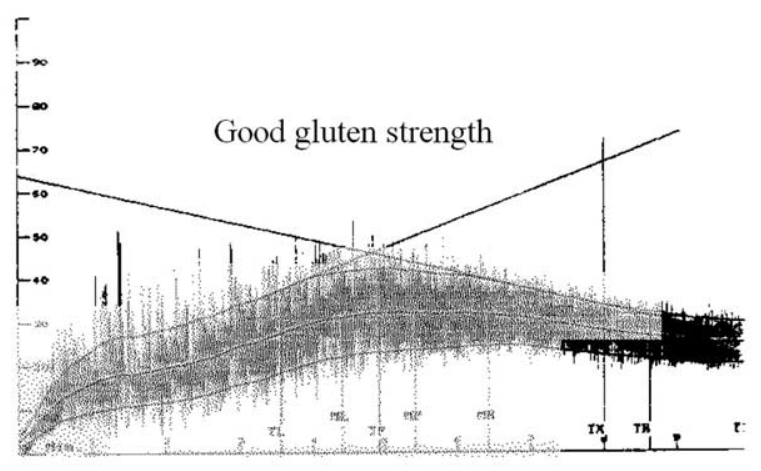

Klasic Wheat Flour

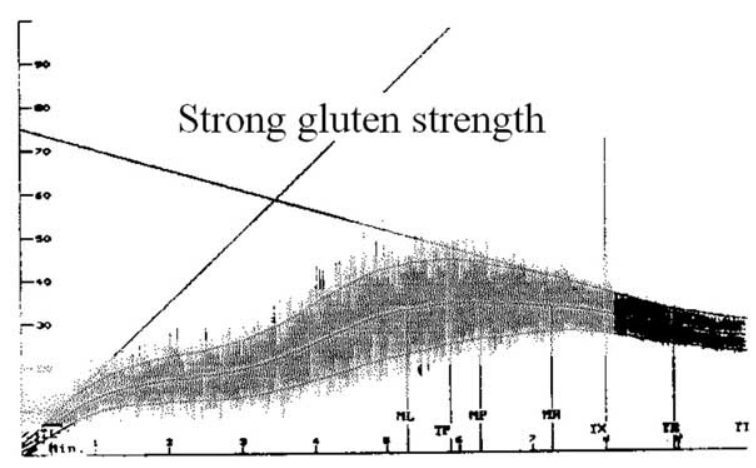

Glenlea Wheat Flour

Fig. 1. Mixograph curves of Chinese black-grained wheat flour compared to its controls in water. 


\section{3.4. Mixograph characteristics}

365 The mixograph results for black-grained wheat flour 366 and its controls are summarized in Fig. 1 and Table 4.

367 Difference in mixograph curves also indicated good or 368 poor gluten strength of different wheat cultivars 369 (Fig. 1). Gluten 'strength' could be estimated from mix370 ograph curve and some of the mixograph parameters. 371 Of the mixograph parameters, midline peak time 372 (MPT) and integral at MPT (MPTI) showed the best 373 correlation with gluten strength. Height value at MPT 374 (MPTH) showed the best relationship with baking per375 formance of gluten and with loaf volume for unfraction376 ated and reconstituted flours. Khatkar and Schofield 377 (1996) indicated that MPTH (also known as peak dough

Table 4

Comparison of mixograph characteristics of wheat flours

\begin{tabular}{|c|c|c|c|c|}
\hline & MPT (min) & $\begin{array}{l}\text { MPTI } \\
\left(\% \mathrm{Tq}^{* \min }\right)\end{array}$ & $\begin{array}{l}\text { MPTH } \\
\text { (MU) }\end{array}$ & $\begin{array}{l}\text { MPTW } \\
\text { (MU) }\end{array}$ \\
\hline \multicolumn{5}{|l|}{$B G W$-flour } \\
\hline In water & $2.93 \mathrm{e}$ & $67.1 \mathrm{~d}$ & $33.6 \mathrm{~b}$ & $16.1 \mathrm{~b}$ \\
\hline In $1 \% \mathrm{NaCl}$ & $4.37 \mathrm{~b}$ & $106.8 \mathrm{~b}$ & $36.4 \mathrm{~b}$ & $19.0 \mathrm{~b}$ \\
\hline In $2 \% \mathrm{NaCl}$ & $3.90 \mathrm{c}$ & $106.6 \mathrm{~b}$ & $41.8 \mathrm{a}$ & $26.2 \mathrm{a}$ \\
\hline In $3 \% \mathrm{NaCl}$ & $5.03 \mathrm{a}$ & $148.9 \mathrm{a}$ & $42.4 \mathrm{a}$ & $27.4 \mathrm{a}$ \\
\hline In $1 \%$ Sucrose & $3.52 \mathrm{~d}$ & $92.5 \mathrm{c}$ & $37.2 \mathrm{~b}$ & $19.7 \mathrm{~b}$ \\
\hline LSD & 0.4561 & 11.0315 & 4.2358 & 3.7351 \\
\hline \multicolumn{5}{|l|}{$T W$-flour } \\
\hline In water & $2.26 \mathrm{ab}$ & $46.2 b$ & $32.3 b$ & $15.8 \mathrm{~b}$ \\
\hline In $1 \% \mathrm{NaCl}$ & $2.13 b c$ & $48.3 b$ & $35.3 b$ & $19.7 \mathrm{a}$ \\
\hline In $2 \% \mathrm{NaCl}$ & $2.44 \mathrm{a}$ & $64.8 \mathrm{a}$ & $40.3 \mathrm{a}$ & $22.0 \mathrm{a}$ \\
\hline In $3 \% \mathrm{NaCl}$ & $2.26 \mathrm{ab}$ & $45.5 b$ & $32.6 \mathrm{~b}$ & $22.6 \mathrm{a}$ \\
\hline In $1 \%$ Sucrose & $1.89 \mathrm{c}$ & $40.9 \mathrm{~b}$ & $32.2 b$ & $14.8 b$ \\
\hline LSD & 0.3016 & 7.936 & 4.1052 & 3.5813 \\
\hline \multicolumn{5}{|l|}{$K W$-flour } \\
\hline In water & $5.41 \mathrm{~d}$ & $122.1 \mathrm{~d}$ & $32.3 \mathrm{c}$ & $17.9 \mathrm{c}$ \\
\hline In $1 \% \mathrm{NaCl}$ & $5.93 \mathrm{c}$ & $135.4 \mathrm{c}$ & $36.6 \mathrm{~b}$ & $23.3 b$ \\
\hline In $2 \% \mathrm{NaCl}$ & $6.76 \mathrm{~b}$ & $170.5 b$ & $42.4 \mathrm{a}$ & $28.8 \mathrm{a}$ \\
\hline In $3 \% \mathrm{NaCl}$ & $7.76 \mathrm{a}$ & $211.8 \mathrm{a}$ & $42.4 \mathrm{a}$ & $25.9 \mathrm{ab}$ \\
\hline In $1 \%$ Sucrose & $5.53 \mathrm{~d}$ & $142.8 \mathrm{c}$ & $37.8 \mathrm{~b}$ & $20.0 \mathrm{c}$ \\
\hline LSD & 0.3952 & 12.3841 & 4.2762 & 3.2018 \\
\hline \multicolumn{5}{|l|}{$Y R W$-flour } \\
\hline In water & $6.11 \mathrm{~d}$ & $115.3 \mathrm{c}$ & $27.2 b$ & $14.2 \mathrm{c}$ \\
\hline In $1 \% \mathrm{NaCl}$ & $6.69 c$ & $130.7 b$ & $30.0 \mathrm{~b}$ & $18.7 \mathrm{~b}$ \\
\hline In $2 \% \mathrm{NaCl}$ & $7.21 \mathrm{ba}$ & $151.6 \mathrm{a}$ & $34.8 \mathrm{a}$ & $21.5 \mathrm{ba}$ \\
\hline In $3 \% \mathrm{NaCl}$ & $7.57 \mathrm{a}$ & $155.7 \mathrm{a}$ & $36.5 \mathrm{a}$ & $24.7 \mathrm{a}$ \\
\hline In $1 \%$ Sucrose & $7.06 \mathrm{cb}$ & $122.7 \mathrm{cb}$ & $27.7 b$ & $13.3 \mathrm{c}$ \\
\hline LSD & 0.4361 & 13.8255 & 4.6139 & 3.8013 \\
\hline \multicolumn{5}{|l|}{$G W$-flour } \\
\hline In water & $6.27 \mathrm{~b}$ & $134.8 \mathrm{~b}$ & $34.4 \mathrm{bc}$ & $17.5 b$ \\
\hline In $1 \% \mathrm{NaCl}$ & $5.63 \mathrm{c}$ & $101.5 \mathrm{c}$ & $30.2 \mathrm{~d}$ & $18.4 \mathrm{~b}$ \\
\hline In $2 \% \mathrm{NaCl}$ & $6.97 \mathrm{a}$ & $139.6 \mathrm{~b}$ & $37.9 \mathrm{ba}$ & $24.7 \mathrm{a}$ \\
\hline In $3 \% \mathrm{NaCl}$ & $7.22 \mathrm{a}$ & $150.4 \mathrm{a}$ & $40.6 \mathrm{a}$ & $26.1 \mathrm{a}$ \\
\hline In $1 \%$ Sucrose & $6.30 \mathrm{~b}$ & $126.4 \mathrm{~b}$ & $31.9 \mathrm{dc}$ & $17.0 \mathrm{~b}$ \\
\hline LSD & 0.5137 & 13.6913 & 3.9016 & 3.3306 \\
\hline
\end{tabular}

MPT - M, mid line; P, peak; $T$, time (min). MPTI - integral $(\%$ Tq*min) at MPT. MPTH - height value (MU) at MPT. MPTW width (MU) at MPT. LSD - least significance difference at $p<0.05$ level of probability. Mean values for individual flours having similar letters in the same column are not significantly different. resistance) was the most useful mixograph parameters for assessing gluten bread making quality, and the $2 \mathrm{~g}$ mixograph proved to be a simple and rapid instrument for studying mixing properties and for evaluating the baking potential of gluten. Weak gluten developed quickly and needed a shorter time to mix to MPTH. A longer time period to mix to MPTH indicated better gluten strength in the wheat flour. A longer duration was also consistent with its relatively high MPTI value. In water, gluten strength for BGW flour (MPT $2.93 \mathrm{~min}$ ) was weak when compared to KW, YRW and GW flours $(\mathrm{MPT}>5.00 \mathrm{~min})$. Salt and sucrose had significant effects $(p<0.05)$ on gluten strength of BGW flour and the controls (Table 7). He, Roach, and Hoseney (1992) reported that neutral salts could change the hydrophobic interaction among gluten proteins and that the gluten proteins from poor-quality flour were less hydrophobic than those from the good-quality flour. Our results indicated that the effect of salt on gluten was associated with its strength. For relatively strong glutens, as found in BGW, KW, YRW and GW flours, adding salt further increased the strength. However, for TW flour containing weak gluten, adding salt did not affect its gluten strength. The effect of sucrose on gluten was also associated with gluten strength. For BGW flour, a $1 \%(\mathrm{w} / \mathrm{v})$ sucrose solution significantly increased MPT $(p<0.05)$. In contrast, the same solution decreased MPT by $0.37 \mathrm{~min}$ in TW flour. The effects of salt and sucrose on MPTH value (used in predicting loaf volume) varied with different wheat cultivars and require further studies.

\subsection{Dough stickiness}

Results on dough stickiness of six wheat flour samples are listed in Table 5. Dough stickiness decreased in flours as follows: $\mathrm{AW}>\mathrm{TW}>\mathrm{YRW}>\mathrm{BGW}>$ $\mathrm{KW}>\mathrm{GW}$. Dough stickiness has an important effect on bakery characteristics because sticky doughs present problems during baking (Chen \& Hoseney, 1995). Hence bakery characteristics of Chinese black-grained wheat flour were significantly better $(p<0.05)$ than that of YRW, TW and AW flours, but somewhat stickier in comparison with $\mathrm{KW}$ and GW flours.

\subsection{Electrophoretic storage-protein profile}

The SDS-PAGE electrophoregrams of total storage protein extracted from the BGW, TW, KW, YRW, GW and AW are presented in Fig. 2. In the high-molecular-weight glutenin (HMW-glu) region, bands (genotype code) appearing at the top for YRW and AW and GW have been reported before. Genes have, respectively, coded the $1,17+18$ and $5+10$ subunits in Yecora Roji (Mansur, Qualset, Kasarda, \& Morris, 1990; Martin \& Carrio, 1999), the $2+12$ and $7+8$ 
W. Li et al. / Food Chemistry $x x x$ (2005) $x x x-x x x$

Table 5

Dough stickiness characteristics

\begin{tabular}{llllllll}
\hline Name & BGW-flour & TW-flour & KW-flour & YRW-flour & GW-flour & AW-flour & LSD \\
\hline Stickiness $(\mathrm{g})$ & $223.76 \mathrm{~d}$ & $392.75 \mathrm{~b}$ & $186.01 \mathrm{e}$ & $313.05 \mathrm{c}$ & $182.67 \mathrm{e}$ & $414.49 \mathrm{a}$ & 21.3041 \\
\hline
\end{tabular}

LSD, least significance difference at $p<0.05$ level of probability. Mean values for flour samples having similar letters in the same row are not significantly different.

430 subunits in Anza (Carrillo, Rousset, Qualset, \& Kasar431 da, 1990; Mansur et al., 1990) and the $2^{*}, 7+8$, and $4325+10$ subunits in Glenlea (Hussain, Lukow, \& McKen433 zie, 1998; Kim \& Bushuk, 1995). Our results showed 434 that bands for Taifen 1 wheat were similar to those of 435 Anza wheat whereas bands for Klasic wheat were simi436 lar to those of Yecora Rojo wheat in the HMW-glu re437 gion. Bands appearing at the top for the Chinese black438 grained wheat were similar to those of Glenlea wheat. 439 Results also showed that some bands for BGW and $440 \mathrm{GW}$ were similar to those of KW and YRW whereas 441 other bands for BGW and GW were similar to those 442 of TW and AW. Many studies (Bournouf \& Bouriouet, 443 1980; Moonen, Kescheepstra, \& Graveland, 1982; 444 Payne, Holt, Harinder, McCartney, \& Lawrence, 1987) 445 have proved that baking quality is strongly correlated 446 with the presence or absence of HMW-glu subunits 1 447 and $2^{*}$ and subunits 5 and 10 . Poor baking quality is 448 usually associated with subunits 2 and 12. SDS-PAGE 449 result of BGW was useful for predicting its potential 450 use. Band 2* subunit for BGW and GW was not very

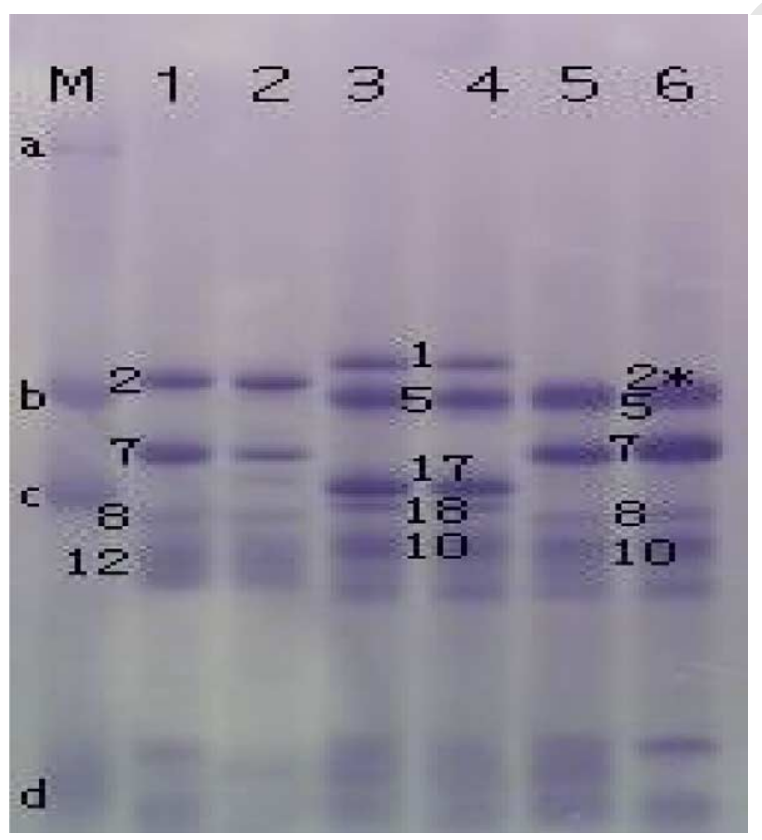

Fig. 2. $10 \%$ SDS gel electrophoresis profile of black-grained wheat protein compared to five wheat controls. Notes. $\mathrm{M}-$ molecular weight marker ((a) $205 \mathrm{kDa}$; (b) $116 \mathrm{kDa}$; (c) $66 \mathrm{kDa}$; (d) $39.8 \mathrm{kDa}$ ); 1 - Anza wheat (genotype code: $2,7,8,12$ ); 2 - Taifen 1 wheat (genotype code: same as Anza); 3 - Klasic wheat (genotype code: same as Yecora Rojo); 4 - Yecora Rojo wheat (genotype code: 1, 5, 17, 18, 10); 5 Black-grained wheat (genotype code: same as Glenlea); 6 - Glenlea wheat (genotype code: $2^{*}, 5,7,8,10$ ). clear under this electrophoretic conditions and further studies are needed.

\section{Conclusions}

Protein properties of Chinese black-grained wheat were evaluated in comparison with five specially selected wheat controls. Gluten index and mixograph data indicated that gluten strength in Chinese black-grained wheat flour was better than Taifen 1, but significantly poorer $(p<0.05)$ when compared to Klasic, Yecora Rojo and Glenlea wheat flours. Dough stickiness data showed that Chinese black-grained flour was significantly weaker $(p<0.05)$ than that of Anza, Taifen 1 and Yecora Rojo, but stronger $(p<0.05)$ in comparison to Klasic and Glenlea wheat flours. Because of the high protein content in Chinese black-grained wheat whole meal and its flour, the total essential amino acid and total amino acid contents were also higher than levels found in controls. After high temperature drying of wet gluten, amino acid composition of Chinese blackgrained wheat gluten was heat stable with minimal loss in comparison with Taifen 1 and Klasic wheat gluten. With a high content of elemental Se and protein and possibly phenolic compounds, Chinese black-grained wheat is potentially a raw material for the development of functional foods. It appears as a suitable candidate for noodle production and in bread-making if used in combination with strong flours. Its gluten strength can be further improved through breeding.

\section{Acknowledgements}

479

This research project was supported by grants from the Hong Kong Research Grants Council and the University of Hong Kong Committee on Research and Conference Grants. We thank the Shanxi Scholarship Council on Research Grants, the Shanxi Natural Science Fund, the Shanxi Education Office on Research Grants, and Shanxi Science \& Technology Office on Research Grants for support.

\section{References}

AACC. (1995). Approved methods: 9th ed. Methods 38-12. St. Paul, MN: American Association of Cereal Chemists. 
AACC. (1995). Approved methods: 9th ed. Methods 46-11A. St. Paul, $\mathrm{MN}$ : American Association of Cereal Chemists.

AACC. (1995). Approved methods: 9th ed. Methods 54-40A. St. Paul, MN: American Association of Cereal Chemists.

Abdel-Aal, E. S. M., Hucl, P., \& Sosulski, F. W. (1995). Composition and nutritional characteristics of spring einkorn and spelt wheats. Cereal Chemistry, 72, 621-624.

Acouistucci, R., Degidio, M. G., \& Vallega, V. (1995). Amino acid composition of selected strains of diploid wheat, Triticum monococcum L. Cereal Chemistry, 72, 213-216.

Anjuma, F. M., Ahmada, I., Butta, M. S., Sheikhb, M. A., \& Pasha, I. (2005). Amino acid composition of spring wheats and losses of lysine during chapati baking. Journal of Food Composition and Analysis, 18, 523-532.

Awika, J. M., Rooney, L. W., \& Waniska, R. D. (2004). Anthocyanins from black sorghum and their antioxidant properties. Food Chemistry, 90, 293-301.

Bai, Y. F., Hou, B. Y., Sun, S. C., Sun, Y., Pei, Z. Y., \& Yan, G. Y. (2002). Genetic differences of gliadin profiles of black-grained wheat germplasm resources. Journal of Triticeae Crops, 22(2), 22-25 (in Chinese).

Bai, Y. F., Li, W. D., Sun, S. C., Sun, Y., Pei, Z. Y., \& Corke, H. (2000). Evaluation on some quality characteristics of black-grained wheat 76. Journal of the Chinese Cereals and Oils Association, 15(2), 6-9 (in Chinese).

Beta, T., Nam, S., Dexter, J. E., \& Sapirstein, H. D. (2005). Phenolic content and antioxidant activity of pearled wheat and roller mill fractions. Cereal Chemistry, 82, 390-393.

Bournouf, T., \& Bouriouet, R. (1980). Glutenin subunits of genetically related European hexaploid wheat cultivars: Their relation to bread-making quality. Theoretical and Applied Genetics, 58, 107-111.

Carrillo, J. M., Rousset, M., Qualset, C. O., \& Kasarda, D. D. (1990). Use of recombinant inbred lines of wheat for study of associations of high-molecular-weight glutenin subunit alleles to quantitative traits 1. Grain yield and quality tests. Theoretical and Applied Genetics, 79, 321-330.

Chen, W. Z., \& Hoseney, R. C. (1995). Development of an objective method for dough stickiness. Lebensmittel Wissenschaft und Technologie, 28, 467-473.

Dick, J. W., \& Quick, J. S. (1983). A modified screening test for rapid estimation of gluten strength in early-generation durum wheat breeding lines. Cereal Chemistry, 60, 315-318.

Fujino, H., Muguruma, M., Mori, K., Tsueno, D., Sasaki, A., Ito, T., et al. (1995). Effect of preheating at high temperature under vacuum on heat aggregation of whey protein isolate (studies on available utilization of whey proteins: Part III). Journal of the Japanese Society for Food Science and Technology, 42(10), 756-761.

Fullington, J. G., Cole, E. W., \& Kasarda, D. D. (1983). Quantitative SDS-PAGE of total proteins from different wheat varieties: Effects of protein content. Cereal Chemistry, 60, 65-70.

Gopal, D. H., Monteiro, P. V., Virupaksha, T. K., \& Ramachandra, G. (1988). Protein concentrates from Italian millet (Setaria italica) and their enzymatic hydrolysis. Food Chemistry, 29, 97-108.

He, H., Roach, R. R., \& Hoseney, R. C. (1992). Effect of nonchaotropic salts on flour bread-making properties. Cereal Chemistry, 69, 366-371.

Hussain, A., Lukow, O. M., \& McKenzie, R. I. H. (1998). Rheological properties of gluten derived from wheat cultivars with identical HMW glutenin subunit composition. Journal of the Science of Food and Agriculture, 78(4), 551-558.

Khatkar, B. S., \& Schofield, J. D. (1996). Assessment of wheat-gluten quality using the $2 \mathrm{~g}$ direct-drive Mixograph. In C. W. Wrigley (Ed.), Gluten' 96. Proceedings of the 6th international gluten workshop (pp. 511-514). Royal Australia Chemistry Institute: Melbourne.
Kim, H. R., \& Bushuk, W. (1995). Salt sensitivity of acetic acidextractable protein of wheat flour. Journal of Cereal Science, 21, 241-250.

Kruger, J. E., \& Reed, G. (1988). Enzymes and color (third ed.. In Y. Pomeranz (Ed.). Wheat: chemistry and technology (vol. I, pp. 441-500). St. Paul: American Association of Cereal Chemists.

Laemmli, U. K. (1970). Cleavage of structural proteins during the assembly of the head of the bacteriophage T4. Nature (London), $227,680-685$.

Lai, L. Z. (1995). Introduction regarding black food. In L. Z. Lai (Ed.), Study on black foods (pp. 2-6). Beijing: China Agricultural Publishing House.

Lai, L. Z., \& Zhang, M. W. (1995). Research and manufacture of black glutinous coarse rice flour. Guangdong Agricultural Science, 5, 24-25 (in Chinese).

Li, W. D., Corke, H., \& Sun, S. C. (1998). Some characteristics of Chinese black-grained wheat flour. In H. Corke \& R. Lin (Eds.), Asian food product development (pp. 76-82). Beijing: Science Press.

Li, W. D., Sun, S. C., \& Ren, G. X. (2004). Study on characteristic of black wheat and its exploitation and utilization. China Condiment, 1, 9-11, 26 (in Chinese).

Llames, C. R., \& Fontaine, J. (1994). Determination of amino acids in feeds: Collaborative study. Journal AOAC International, 77, 1362-1402.

Mansur, L. M., Qualset, C. O., Kasarda, D. D., \& Morris, R. (1990). Effect of 'Cheyenne' chromosomes on milling and baking quality in 'Chinese Spring' wheat in relation to glutenin and gliadin storage proteins. Crop Science, 30, 593-602.

Martin, P., \& Carrio, J. M. (1999). Cumulative and interaction effects of prolamin allelic variation and of 1BL/1RS translocation on flour quality in bread wheat. Euphytica, 108, 29-39.

Moonen, J. H., Kescheepstra, A. U., \& Graveland, A. (1982). Use of the SDS-sedimentation test and SDS-polyacrylamide electrophoresis for screening breeder's samples of wheat for bread-making quality. Euphytica, 31, 677-690.

Payne, P. I., Holt, L. M., Harinder, K., McCartney, D. P., \& Lawrence, G. J. (1987). The use of near-isogenic lines with different HMW glutenin subunits in studying bread-making quality and glutenin structure. In R. Lasztity \& F. Bekes (Eds.), Proceedings of the 3rd international workshop on gluten proteins (pp. 216-227). World Scientific: Singapore.

Perten, H. (1990). Rapid measurement of wet gluten quality by the gluten index. Cereal Foods World, 35(4), 401-402.

Qiu, J. W. (1998). Nutrition value and extraction method of wheat gluten. Science and Technology of Food Industry, 2, 56-57 (in Chinese).

Ramachandra, G., Virupaksha, T. K., \& Shadaksharaswamy, M. (1977). Relationship between tannin levels and in vitro protein digestibility in finger millet (Eleusine coracana Gaertn). Journal of Agricultural and Food Chemistry, 25, 1101-1104.

Rath, C. R., Gras, P. W., Wrigley, C. W., \& Walker, C. E. (1990). Evaluation of dough properties from two grams of flour using the mixograph principle. Cereal Foods World, 35, 572-574.

Sun, Q., Sun, B., \& Wang, J. (2004). Characters of seed pigment of black kernel wheat. Seed, 23(6), 19-23 (in Chinese).

Sun, S. C., Sun, Y., Yuan, W. Y., Yan, W. Z., Pei, Z. Y., \& Zhang, M. R. (1999). Breeding and qualitative analysis for black wheat 76 of superior quality. Acta Agronomica Sinica, 25(1), 51-54 (in Chinese).

Sun, S. C., Yuan, W. Y., Liu, S. X., Wang, H., Sun, Y., Zhang, M. R., et al. (1996). A study on the production of tetrageneric hybrids crossed between Triticum, agropyron, haynaldia, secale, and their morphology and cytology. Chinese Journal of Genetics, 23(2), 131-153 (in Chinese).

Yang, J. H., Li, W. D., Chu, X. N., \& Sun, S. C. (2001). Study on black seed wheat soy sauce with nutrition and health function. China Brewage, 2, 31-32 (in Chinese). 